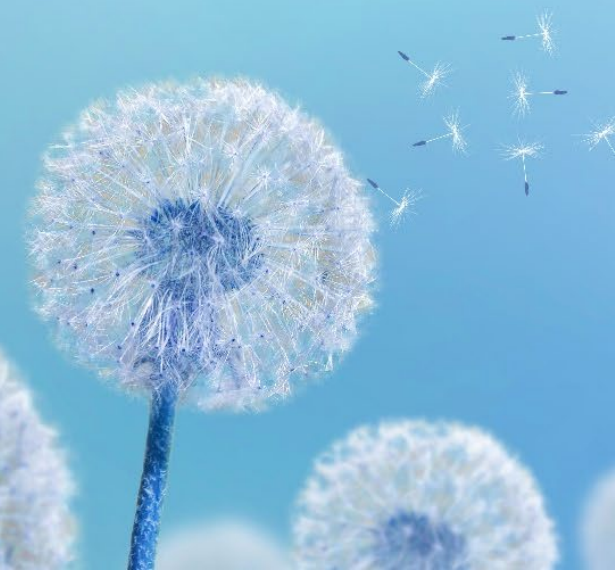

\title{
Adaptation of Trauma-Focused Cognitive Behavioural Therapy for cases of child sexual abuse with complex trauma: A clinical case illustration
}

\section{Martine HÉBERT' ${ }^{1}$, Isabelle V. DAIGNAULT ${ }^{2}$, and Claudia BLANCHARD-DALLAIRE ${ }^{3}$}

\footnotetext{
1 Canada Research Chair in Interpersonal Trauma and Resilience

2 École de criminologie, Université de Montréal

3 Fondation Marie-Vincent
}

Corresponding Author: Martine Hébert, Ph.D., Département de sexologie, PO Box 8888 succursale Centre-Ville, Montréal, Québec, Canada H3C $3 \mathrm{P} 8$.

Email: hebert.m@uqam.ca.

\section{Abstract}

Child sexual abuse is an important public health issue given its magnitude and the multiple associated consequences. The diversity of profiles in child victims of sexual abuse calls for a more personalized approach to treatment. Indeed, recent studies suggest that children display a variety of symptoms and that a subgroup of sexually abused children may present a profile of complex trauma. This article first presents a review of the scientific literature that positions Trauma-Focused Cognitive Behavioural Therapy (TF-CBT; Cohen et al., 2017) amongst the best practices to address trauma-related symptoms following child sexual abuse; whether it is co-occurring with other forms of violence or not. Various adaptations of TF-CBT therapy are proposed by the authors (Cohen et al., 2012) to treat children facing complex trauma. These adaptations are summarized and illustrated with the presentation of a clinical case involving two siblings from the same family.

Keywords: Child sexual abuse; complex trauma; therapy; case study. 


\section{Introduction}

Child sexual abuse (CSA) is a major public health issue with global prevalence estimates revealing that between $8 \%$ to $31 \%$ of girls and $3 \%$ to $17 \%$ of boys have been victims of sexual violence before the age of 18 (Barth et al., 2013). Reviews of the scholarly literature have highlighted the wide variety of consequences associated with CSA and the relevance of identifying factors associated with outcomes in order to design efficient interventions promoting recovery. Among such factors is the frequent co-occurrence of other forms of violence; which studies have commonly related to the presence of multiple factors of adversity in the sexually abused child's family environment (Assink et al., 2019). In fact, child victims of sexual abuse are identified as particularly vulnerable to further victimization in different contexts (Finkelhor et al., 2007), including peer victimization in school (Tremblay-Perreault et al., 2017), dating victimization (Hébert et al., 2017) and cyber-victimization (Hébert et al., 2016).

With regard to consequences, research over the past 30 years reveals that victims of CSA are likely to present with significant internalized disorders (symptoms of anxiety and depression, somatic disorders, withdrawn behavior) as well as externalized disorders (aggressive behavior, anger, conduct disorders) (Lewis et al., 2016). Their functioning in schools is also frequently hampered by impairments in attention and concentration as well as social difficulties (Blanchard-Dallaire \& Hébert, 2014). Further, such consequences in victims exposed to multiple forms of violence have been described as exacerbated (Aho et al., 2016).

While a vast array of symptoms has been linked to CSA, for many authors, sexual abuse and its subsequent reactions are most consistent with the criteria for post-traumatic stress disorder (PTSD). Diagnostic criteria involve the individual experiencing an event that poses a serious threat in which he or she may have been seriously injured or threatened with death or serious injury, or that he or she is threatened or sexually abused. According to DSM-5, four groups of symptoms are evident: symptoms of intrusion, avoidance of trauma-related stimuli, negative impairment of cognition and mood, and marked changes in activation and responsiveness to the traumatic event (e.g., hypervigilance). However, as CSA victims are frequently exposed to other forms of violence, authors have argued that more chronic traumatic situations experienced at an early age could lead to developmental alterations that go well beyond symptoms of classic PTSD. Thus, the classic formulation of PTSD would fail to identify the constellation of difficulties experienced by children who were exposed to repetitive and chronic violence in an inadequate family environment (van der Kolk et al., 2009).

\section{Complex trauma}

The concept of complex trauma in children describes their exposure to traumatic events and the consequences of this exposure on their short- and long-term adaptation (Cook et al., 2003). Children may experience traumatic events involving parenting figures while being victims of other forms of abuse (e.g., sexual assault and witnessing domestic violence). As many children experience co-occurring abuse (Adams et al., 2016), empirical evidence suggests that polyvictimization may be the key element that predicts the intensity of symptoms, or even overshadow the influence of an isolated form of victimization (Finkelhor et al., 2007; Hodges et al. 2013).

Thus, multiple traumatic experiences, loss of sense of security and lack of comforting and supportive parenting figures may increase the risk of further trauma and deterioration in several areas beyond the classic symptoms of PTSD; alterations in relationships with others (e.g., social isolation, relationship difficulties, difficulties in relation to attachment), somatic regulation (e.g., medical problems, hypersensitivity, somatization), emotional regulation (e.g., difficulty recognizing and managing emotions), dissociation (e.g., alterations in states of consciousness), regulation of behaviour (e.g., poor impulse control, aggression), cognition (e.g., learning difficulties and deficits in the executive function) and self-concept (e.g., low self-esteem, feelings of guilt and shame).

This constellation of more complex symptomatology appears to be relevant to the clientele of sexually abused children. One recent analysis in fact identifies three distinct profiles including children showing symptoms of classic PTSD and children displaying a resilient profile (Hébert \& Amédée, 2019). In addition, close to one out of four treatmentseeking sexually abused children are showing a third profile: very high classic PTSD symptoms as well as difficulties in several domains related to complex PTSD, including self-esteem difficulties, cognitive problems, difficulties in regulating emotions and behaviours, somatic symptoms, and symptoms of dissociation (Hébert \& Amédée, 2019).

The existence of various profiles supports the idea that a continuum of interventions must be considered in order to adequately address the varied needs of children. Complex trauma in fact calls for a form of therapeutic 
flexibility on the part of clinical teams that allows for individual adaptations to the specific, yet numerous current manifestations of trauma in the client's life.

\section{The Trauma-Focused Cognitive Behavioural Approach}

TF-CBT is described as a therapeutic approach that focuses on helping children and adolescents learn strategies and coping skills to overcome their traumatic experience and its consequences. "Trauma-focused" specifies that this therapeutic approach involves clearly addressing the occurrence of trauma, as well as the cognitions, sensations and emotions that are associated to its memory. Theoretically grounded in cognitive behavioral theory, this approach more specifically lies on learning theories; according to which the appearance of trauma symptoms can be explained by classical conditioning (Cohen et al., 2006); while operative conditioning (or instrumental learning) can reinforce maintenance of these symptoms and of various reactive behaviours. This therapeutic approach is composed of different modules to be addressed with both the child and his or her parent while planning an increasing number of joint sessions. Trauma reminders and consequences are addressed gradually, at different levels of intensity, depending on the child's and family's needs and capacity. While TF-CBT was initially elaborated in the mid 1980s with the combined works by Judith Cohen and Anthony Mannarino in Pittsburg and Esther Deblinger in New Jersey, at this time, there were no published studies addressing the efficacy of interventions for children who were exposed to traumatic experiences and for those suffering from PTSD. By the late 1990s, Cohen, Mannarino and Deblinger began publishing their first evaluative studies of these interventions, indicating in various samples of children and adolescents that they were evidenced-based (Cohen et al., 2004; Cohen \& Mannarino, 1998). In 2006, they published their treatment manual: Treating Trauma and Traumatic Grief in Children and Adolescents (Cohen et al., 2006). As research documented the occurrence of complex trauma in children and adolescents, Cohen and her colleagues (2012) proposed an adaptation to complex trauma for TF-CBT that allows to consider the particular challenges of this clientele.

The following section summarizes the efficacy of TF-CBT with children who have been exposed to multiple forms of violence or with children who, in addition to being exposed to multiple victimization, also present a complex trauma profile (i.e., issues in various spheres of adaptation). Then, considering that there may be practical concerns with the applicability of TF-CBT to children and adolescents with a complex trauma profile, the adaptations proposed by Cohen and her colleagues (2012) are summarized, described and discussed with case studies of child victims of CSA.

\section{Effectiveness of TF-CBT with particular cases}

While there are a multitude of evaluative studies on TF-CBT (see for e.g., Runyon et al., 2019), this section highlights studies that have offered information regarding multiple traumas experienced by participants. Since complex trauma is not yet a recognized formal diagnosis in the DSM 5, the way in which it is conceptualized can vary from one study to another. Hence, the few available studies have mainly explored the effects of TF-CBT in children who have experienced several potentially traumatic events, but not necessarily presenting a complex trauma profile.

In one of their evaluative studies of TF-CBT, Cohen and her colleagues (2004) report that children who participated had experienced, on average, 3.6 types of trauma (e.g., exposure to intimate partner violence, physical abuse, mourning). For the authors, this indicates that TF-CBT is associated with positive effects, even in cases where several types of trauma are present. A study led by Jensen and colleagues (2014) evaluated the effectiveness of TF-CBT in a community setting compared with treatment-as-usual (TAU) as a comparison group with youth aged 10 to 18 randomly assigned to the two conditions. On average, participants were exposed to 3.6 traumas including the sudden death or serious illness of a loved one, threats or violence outside of the family context, and physical violence by a family member or sexual abuse. Results showed that TF-CBT was more effective than TAU in reducing symptoms of post-traumatic stress and depression and improving overall mental health. A subsequent study (Jensen et al., 2017) revealed that improvements were maintained in an 18-month follow-up. Bartlett and colleagues (Bartlett et al., 2018) contrasted outcomes of child welfare involved youth following their participation in either TF-CBT, Attachment, SelfRegulation, and Competency (ARC) or Child-Parent Psychotherapy (CPP). Authors reported optimal benefits for youth who received TF-CBT or ARC. As in other studies, TF-CBT was found to be associated with a significant reduction in PTSD symptoms evaluated by self-reports and parent/caregiver reports and behavior problems, as well as an increase of the child's strengths as evaluated by the Child and Adolescent Needs and Strengths - Mental Health (Lyons et al., 1999). Participants reported experiencing a mean of 5.2 types of trauma (different forms of child maltreatment by caregivers, war/terrorism, bereavement, natural disaster, medical illness, serious injury, etc.) again suggesting that TFCBT is efficient in helping children even if they have been exposed to multiple traumas. Similar conclusions were reached in studies conducted in developing countries with samples of children and adolescents exposed to extremely 
difficult and multiple situations. Culturally adapted versions of TF-CBT allowed to significantly reduce the severity of trauma symptoms in youth exposed to violence towards a family member, victims of sexual exploitation, or former child soldiers, reporting experiencing up to 12 different types of trauma (McMullen et al., 2013; Murray et al., 2013; O'Callaghan et al., 2013).

Few published studies have analyzed the effects of TF-CBT by considering not only the multiplicity of traumas experienced, but also the complexity of the symptoms among participants. In one study conducted by Weiner, Schneider and Lyons (2009), the complexity of trauma symptoms was operationalized using the Child and Adolescent Needs and Strengths (CANS; Lyons, 2004). In addition to having had a moderate to severe traumatic experience, participants had to demonstrate difficulties adjusting to trauma, as measured by a score of 2 or 3 on the CANS "trauma adjustment" subscale. TF-CBT was effective in reducing traumatic symptoms in youth. Furthermore, participation in TFCBT also improved the adaptation of youth dealing with complex traumatic symptoms and increased certain skills, as assessed by the CANS "strengths" subscale.

Sachser, Keller and Goldberg (2016) used the diagnostic criteria proposed by the International Classification of Diseases (ICD-11) to identify and distinguish PTSD and a profile more closely related to complex trauma. A latent class analysis identified the two expected profiles: a first group of children presenting the most classic symptoms of PTSD and a second group presenting in addition, symptoms reflecting dysfunctions on the level of emotion regulation, self-concept and interpersonal relationships. The authors then contrasted the evolution of participants undergoing therapy ( $n=38$ for PTSD class and $n=23$ for the Complex trauma class). Results revealed a significant reduction of PTSD symptoms both in participants in the PTSD group and those with a complex trauma profile. In addition, TF-CBT was efficient in reducing impairment in domain characteristics of Complex PTSD (i.e., emotion dysregulation, interpersonal problems and self-concept) in children from the Complex trauma class.

The TF-CBT approach has been adapted and implemented at the Marie-Vincent Foundation (a Child Advocacy Center) in Montreal, Quebec, a centre that offers services to child and adolescent victims of sexual abuse. The effectiveness studies on the adaptation of TF-CBT in Quebec were conducted with a sample of preschool children (Hébert \& Daignault, 2015) and with a sample of children aged 6 to 12 (Hébert \& Tourigny, 2015). Findings showed significant effects in reducing symptoms of dissociation, internalizing and externalizing behavior problems in preschool and school-aged children. For the latter group, other outcomes assessed revealed an improvement in children's posttraumatic stress symptoms, self-esteem and feelings of guilt. More than half (55\%) of the sample of school-aged children experienced more than four traumatic events other than sexual abuse that motivated their request for services. An analysis contrasting the evolution of symptom reduction in children reporting more than four traumas revealed no significant differences in comparison to children with fewer than four traumas. These results suggest that TF-CBT is associated with similar benefits for youth exposed to multiple traumas. In a replication of the study conducted by Sachser et al. (2016), TF-CBT was found to be effective in sustaining recovery even in children presenting with a profile akin to complex PTSD profile (Hébert \& Amédée, submitted). The following case study will illustrate the different adaptations of TF-CBT proposed by Cohen et al. (2012).

\section{Case study presentations and adaptations of TF-CBT (Cohen et al。, 2012)}

The case study was inspired from the clinical caseload of therapists working within the Marie-Vincent Foundation. Personal and factual information were modified to ensure confidentiality.

Lisa, an 11-year-old girl, and Jacob, her 8-year-old brother, grew up with their maternal grandparents. Their father is unknown, and their mother has been unable to provide for their security and development. The environment in which they grew up is characterized by poverty and significant social isolation. Throughout their childhood with their grandparents, the grandfather was suffering from alcoholism and they were exposed to violence in many forms. Their trauma history includes witnessing family violence, being victims of psychological abuse and neglect, as well as severe and frequent physical abuse. Lisa and Jacob were also victims of very severe, frequent and chronic sexual abuse, including exposure to pornography, and they also witnessed each other's abuse. Outside of the home, the siblings were also victims of school bullying in relation to hygiene issues. During the unveiling of the sexual abuse, the children experienced major disturbances. They were first placed in foster care, then moved with their biological mother who has built a second family; hence the reconstruction of the mother-child bond to Jacob and Lisa being an important issue. As they have evolved for years in an environment poorly suited to their needs and having been confronted with many adverse experiences compromising their security and development, both Lisa and Jacob present a worrying 
symptomatic picture, that considering their history of trauma, also mirror the various domains affected in cases of complex trauma.

Lisa (11 years old) is in a constant search for attention and affection. She is impressionable, wants to be loved at all costs and often presents with seductive behaviours (e.g., dance, sexualized poses). She has some memories of the time she lived with her mother, which contributes to her feeling of abandonment. Social situations are difficult for her as she experiences frequent conflicts, rejection, bullying at school as well as on the social network. She is also struggling academically; she has little concentration and trouble with organization and perseverance. She manifests a great need to talk about the various traumas she has experienced, especially the sexual abuse. She does not target the right people to confide in, which leads to further stigmatization. She presents with very severe PTSD symptoms including intrusive and painful reexperiencing symptoms and dissociation. All domains of complex trauma are affected.

As for Jacob (8 years old), it is difficult to gain access to what he thinks or feels. He is described as a boy who stares for long periods of time. He rarely manifests sadness or distress and shows little reaction. On the other hand, he presents with externalized behaviours. He is often in opposition, he does not listen to instructions or ignores them, he is rude and often reacts aggressively. He presents with low self-esteem, receives little appreciation or attention and is described as the "black sheep" of the family. He reports feeling rejected. He is very impulsive and reckless, adopts risky behaviours and seems completely unaware of the potential surrounding dangers. He has experienced serious falls or injuries; his body is constantly covered in bruises with no memories or explanations of how he sustained such injuries. He reports having intrusive thoughts related to self-harm. Jacob also presents with sexual behaviours (verbalizations of a sexual nature, rubbing one's sexual parts on objects, sound with a sexual connotation). He has great social difficulties. He describes having no friends, being laughed at, being in conflict with others and evokes that no one showed up to his birthday party. He is described as having few social skills and as being unable to decode social situations. Academically, he is experiencing failures and forgetfulness. Teachers describe that he lacks learning availability despite good intellectual potential and that he is often regressed. He also presents with very severe PTSD symptoms including dissociation. All domains of complex trauma are affected.

In describing their proposed adaptation to children with complex trauma, Cohen and her colleagues (2012) argue that similarly to other treatment programs elaborated for complex trauma (Ford \& Courtois, 2009; Lanktree \& Briere, 2017), the components of intervention of the standard TF-CBT are phase-based. In the following section, each of the phases is first described generally, followed by the presentation of adaptations that may be integrated within each phase of the clinical illustrated.

The first part of the therapeutic process comprises a "stabilization phase". It involves acquiring coping strategies for regulating emotions and managing the frequent neurobiological effects of trauma that are associated with being exposed to chronic stress. It thus includes developing an understanding of traumatic reactions and identifying and recognizing emotional states and thoughts in relation to trauma reminders. More specifically, during this first phase, TF-CBT components include psychoeducation and acquiring knowledge and skills to enhance safety and help parents develop parenting skills using relaxation methods and cognitive and affective modulation skills. The second part of the therapy is the "trauma processing" phase; which can also include in vivo mastery of trauma reminders. The trauma processing phase is conditional to first acquiring some degree of coping skills acquired within the stabilization phase. It involves gradual exposure to trauma reminders and anxiety-provoking situations through a creative process (for e.g., writing a story, a play, a song, a poem about various aspects of the trauma), restructuring erroneous or harmful thoughts and acquiring problem-solving strategies. The third phase is described as an "integration phase". The TF-CBT components addressed within this last phase include conjoint child and parent sessions to facilitate communication, enhancing safety skills and future development and addressing traumatic grief. More specifically self-regulation skills are consolidated and generalized to various situations and self-protection and assertiveness abilities are exercised. Parents are accompanied so that they can support their child during the therapeutic process in developing effective communication and management of difficult behaviours and in addressing future issues such as sexual education.

In adapting TF-CBT to youth with a complex trauma trajectory, Cohen et al. (2012) formulated recommendations; which we have summarized into five main adaptations, that are integrated within each phase. 


\section{Phase I - Stabilization phase}

1. Extend the stabilization phase (establishing a secure therapeutic relationship; identifying, expressing and regulating emotions).

2. Allocate more time and vigilance to notions of security both within and outside of the therapeutic relationship (extended parenting/caretaker support).

Concerning the stabilization phase of therapy, Cohen et al. (2012) recommend extending this first phase of the therapeutic process compared to what would be advised for a single trauma; specifying that as much as $50 \%$ of the therapeutic process could be dedicated to this phase. In many respects, adapting TF-CBT to complex trauma is a question of timing and dosing the therapeutic steps and processes to the rhythm and capacity of the child. A clinical comprehension is only accessible to the therapist in the context of a safe, empathic, and honest therapeutic relationship and with the collaboration of a parent/caretaker support system. While attributing more time to reach the objectives of this phase often prolongs the therapy, it has three advantages: a) allows children to reach a feeling of security within the therapeutic relationship; b) which also allows children to open up regarding remaining feelings of fear and uncertainty at the beginning of the intervention and through the therapeutic process; c) allows more time to develop and practice coping and self-regulation while carefully monitoring and "calibrating" distress tolerance in the child and caregiver.

The stabilization phase for Jacob. For Jacob, the context and nature of the therapeutic relationship seemed to be perceived as threatening. Thus, his therapist had to gradually expose Jacob to the idea of trusting again, but this time in the context of a safe and predictable relationship. For children like Jacob, feeling safe again may involve learning about how the brain has a tendency to generalize fear responses for survival, and to distinguish unreal from real dangers and situations. While Jacob's coping skills for reacting to real dangers were over-developed (e.g., being distrustful, oppositional or rude), those for recognizing and making good use of safe situations remained a challenge. This was reflected to him in order for Jacob to develop social awareness, to appease some distress and learn to benefit from the safe situations he experiences. Jacob also developed at-risk behaviours that may have helped him feel more control over his fears. Yet, this process had to be addressed in order to help Jacob learn self-calming or suiting behaviours to replace those that were putting him at risk. In Jacob's case, another strategy that helped establish a bond of trust included following a strict therapeutic routine that became predictable and thus promoted a feeling of security. Feeling safe in various contexts also involved developing Jacob's social adaptation in different situations. Learning to identify and recognize emotions more efficiently is often a prerequisite for self-regulation in social situations, as it was the case for Jacob. Yet, for some children like Jacob, a certain detachment or a limited sense of self-awareness proved to be an important survival strategy, especially when violence was experienced on a recurring basis. Again, Jacob needed to differentiate real from anticipated dangers and to practice using appropriate strategies in social contexts. The following summary box presents a list of additional strategies that may be used with both Lisa and Jacob during the first phase of therapy.

Other family-oriented strategies that may be integrated for Lisa and Jacob

- The first session with Lisa and Jacob could take place in their current living environment for their mother to model trust toward the therapist and transparency.

- After obtaining children's approval to share a specific information with the mother, the therapist should be very transparent in the exchange of information concerning the children (with whom the therapist speaks, meets, etc.) in order to foster a feeling of trust and control.

- Promote the participation of the mother by planning an increasing number of joint sessions to help consolidate a relationship that is based on security, understanding, trust and respect.

- Increase the mother's sensibility to the needs expressed by the problematic behavior of Jacob as he is often perceived as being oppositional and provocative (modulate the mother's perception so that she sees Jacob's difficulties as being possibly associated with distress and with the difficult experiences of his early childhood). 
Other individual therapeutic strategies that may be integrated for Lisa and Jacob

- Plan shorter therapy sessions for their first meetings and make the session plan accessible and predictable for the children in order to establish a routine, reassure them on the themes addressed and allow them to make choices.

- Establish coherent intervention/messages/strategies in the various living environments of the children (home, school, therapy, etc.).

- Dedicate several sessions to the development of self-regulatory strategies by referring to situations that have occurred during the week.

- To facilitate the acquisition of grounding skills, adapt relaxation strategies to more physically based exercises (e.g., yoga, dance) rather than meditation or visualization.

- Use the situations that arose during meetings with the therapist to do "modelling" in relation to self-regulatory strategies.

- Be attentive to Lisa and Jacob's non-verbal reactions, help them recognize their reactions, emotions and thoughts, normalize them and help them feel better.

- For Jacob, develop other means of communication/exchange (e.g., pointing, choice of answers, puppets).

- Help Lisa recognize and identify her need for attention and affection and identify a significant adult she can refer to in different settings to limit the occurrence of risky behaviours that increase the risk of victimization or her feelings of fear.

\section{Phase II - Trauma processing phase}

1. Assign more time to proceed with a slow rhythm for the gradual exposure to traumatic memories (allowing time to associate trauma reminders to emotions).

2. Address traumatic memories by regrouping experiences into relational themes (e.g., feeling unsafe in a relationship, feeling afraid, betrayed, abandoned, different from the others).

Beginning the trauma processing phase requires for children to use acquired strategies to tolerate distress and self-regulate. Hence, before beginning gradual exposure to memories or trauma reminders, it is advised to monitor the actual sense of security of the child, of his or her level of distress and of his or her degree of preparedness. Addressing complex trauma memory entails to leave more time devoted to understanding feelings in relation to memories. For instance, it may require leaving more room to the daily manifestations of trauma reminders or of trauma consequences than to specific souvenirs that are consciously addressed by soliciting memory. The rational for classifying trauma souvenirs into themes is that memories of multiple traumas are often fragmented and not related to specific events or moments in time in the child's memory. Further, victims of complex trauma often describe multiple trauma reminders that can arise anywhere and anytime. Thus, handling such distressing experiences by grouping them may make the task appear more manageable. Further, in comparison to standard gradual exposure strategies, this approach allows to avoid asking children to choose which of their traumatic experiences is most important or distressful to them to establish an exposure hierarchy. A task, which in itself, is most probably extremely confusing and in many instances inconceivable.

The trauma processing phase for Lisa. Lisa presented as overwhelmed by some of her traumatic memories; as she shared them with anyone and in inappropriate times. Thus, the second phase of TF-CBT was a good opportunity for Lisa to understand the rationale of gradual exposure, as a process that should take place in the context of a safe and trusting relationship; so that this context can help Lisa's brain integrate the experience differently, in a way that would allow her to distance herself from the experience. The intent was to help Lisa choose more appropriate times to share her experiences and to persevere in this particular task by making use of the self-regulatory strategies that she had learned to control her reactions, whether they are physiological, cognitive, emotional or behavioral.

In the standard TF-CBT model, for time-managing matters, clinicians are encouraged not to invest too much time in what is described as "the crises of the week", that is to say everyday events that children may address at the start of the sessions and which are not directly related to the therapeutic objectives sought. In the present case, as Lisa was struggling with issues of abandonment and social rejection, her day-to-day social challenges at school were voluntarily addressed to bring up everyday manifestations of trauma and again encourage use of different selfregulatory strategies that may help to ease her current social interactions. Regarding trauma processing, it helped Lisa 
to slowly address her traumas by themes; such as feeling abandoned as it helped her to begin to break down the steps and to categorize what she was feeling; giving her a sense of recognition and control. Further, as the various themes were addressed, her memories, their reminders and her current struggles were perceived in a more integrated manner. The following summary box presents a list of additional strategies that may be used with both Lisa and Jacob during the second phase of therapy.

Other intervention strategies that may be implemented with Lisa and Jacob

- Use more general, less personalized discourse to help Lisa and Jacob normalize certain thoughts, emotions, reactions. E.g., "It often happens that other children who have experienced sexual abuse ..."

- Make sure to frequently assess the level of distress and the symptoms/difficulties that can be activated by the exposure to traumatic memories.

- Address the subject of Jacob's sexual abuse and other traumas through puppetry.

- Take advantage of other opportunities, less threatening for Jacob, to address themes related to sexual abuse. For example, it was less threatening to speak of sexual behaviours occurring at home than to speak of sexual abuse and other traumas. It created a breach to approach the different types of touches and secrets, and to make the link with the other traumas.

- Adapt the form of the "narrative" and lower expectations about its content.

\section{Phase III - Integration of trauma phase}

1. Attributing more time and importance to the notion of traumatic grief and planning more time to end the therapeutic process.

This last phase of the therapeutic process involves communicating about trauma to the parent/caregiver; thereby "testing out" and strengthening the trust relationship with that person. The integration phase thus aims to help children consolidate and generalize what they have learned and acquired as coping mechanism to deal with trauma and to more generally open up to the possibility of trusting again. In this third phase of the therapeutic process, as the parent or caregiver is cognitively and emotionally available, the last step of trauma processing involves for the child to share a part of his experience with a parent. As the joint sessions become more frequent throughout the therapeutic process, uniting the child and caregiver make it possible to discuss the traumas and its remaining consequences together, within a different perspective and that allows to foresee a different future.

The integration phase for Lisa and Jacob. Strengthening the relationship with Lisa's and Jacob's mother was an important challenge. The mother felt overwhelmed by her children's behavior and discipline problems and maintained a rejecting discourse arguing that she has limited time to devote to her children. As this last phase of the therapeutic process also aims to assist the child in the development of a behavioral mode that allows him or her to maintain a feeling of security (e.g., gradually communicating emotions, asking for help and support when necessary, making good relationship choices); it was important to develop a larger support network for the children within the children's environment (e.g., school, extended family, child protection) The caretakers were hence involved in some phases of the therapeutic process. Further, for both Lisa and Jacob the state of their relationship with their mother involved a certain process of mourning that needed to be addressed in the context of individual therapy by asking children to express their feelings about their griefs. The therapists also scheduled a joint sibling session in which they were asked to make a list of elements that they can rely on their mother for support; and a list of other supportive adults on which they can rely for other needs that their mother may not be in a position to meet at this time. For both children, but especially for Lisa, it was also essential to carefully plan the end of the therapeutic process which can also bring back feelings of loss and abandonment. The last summary box presents a list of additional strategies that may be used with both Lisa and Jacob during this last phase of therapy. 
Other intervention strategies that may be integrated with Lisa and Jacob

- Prepare Lisa and Jacob for the end of the therapeutic process in a collaborative way. The goal is to have them experience a positive ending in the context of the therapeutic relation, a success instead of a repetition of the traumas in the context of previous relationships or a relational loss.

- Use the last therapy sessions to help the children build a toolbox, in which they can place images or objects representing all the strategies developed during therapy.

- Space the last therapy meetings.

- Schedule a meeting with all the other professionals involved with Lisa and Jacob in order to ensure continuity in the interventions (by making them aware of the meeting and the content discussed, as well as future references and objectives).

\section{Conclusion}

This article outlined the diversity of profiles in child victims of sexual abuse and its possible association to complex trauma symptomatology. The evolution of our understanding of complex trauma has led to the adaptation of evidence-based therapeutic approaches, such as TF-CBT of which the efficacy has also been supported in recent studies with victims of CSA. Our sibling case illustration has provided tangible examples of how TF-CBT can be adapted to the precise needs of Jacob and Lisa and outlined specific challenges that are often met with this clientele. Future research should hence continue documenting the outcomes of clinical adaptations for children exposed to complex trauma.

\section{Funding}

Marie-Vincent Interuniversity Chair in Child Sexual Abuse.

\section{Conflict of interest}

The authors have no conflict of interest to disclose.

\section{References}

Adams, Z. W., McCauley, J. L., Back, S. E., Flanagan, J., C., Hanson, R. F., Killeen, T. K., \& Danielson, C. K. (2016). Clinician perspectives on treating adolescents with co-occurring post-traumatic stress disorder, substance use, and other problems. Journal of Child \& Adolescent Substance Abuse, 25(6), 575-583. https://doi.org/10.1080/1067828X.2016.1153555

Aho, N., Proczkowska-Björklund, M., \& Svedin, C. G. (2016). Victimization, polyvictimization, and health in Swedish adolescents. Adolescent Health, Medicine and Therapeutics, 7, 89-99. https://doi.org/10.2147/AHMT.S109587

Assink, M., van der Put, C. E., Meeuwsen, M. W., de Jong, N. M., Oort, F. J., Stams, G. J. J., \& Hoeve, M. (2019). Risk factors for child sexual abuse victimization: A meta-analytic review. Psychological Bulletin, 145(5), 459-489. https://doi.org/10.1037/bul0000188

Barth, J., Bermetz, L., Heim, E., Trelle, S., \& Tonia, T. (2013). The current prevalence of child sexual abuse worldwide: A systematic review and meta-analysis. International Journal of Public Health, 58(3), 469-483. https://doi.org/10.1007/s00038-012-0426-1

Bartlett, J. D., Griffin, J. L., Spinazzola, J., Goldman Fraser, J., Noroña, C. R., Bodian, R., Todd, M., Montaga, C., \& Barto, B. (2018). The impact of a statewide trauma-informed care initiative in child welfare on the well-being of children and youth with complex trauma. Children and Youth Services Review, 84, 110-117. https://doi.org/10.1016/j.childyouth.2017.11.015

Blanchard-Dallaire, C., \& Hébert, M. (2014). Social relationships in sexually abused children: Self-reports and teachers' evaluation. Journal of Child Sexual Abuse, 23(3), 326-344. https://doi.org/10.1080/10538712.2014.888123

Cohen, J. A., Deblinger, E., Mannarino, A. P., \& Steer, R. A. (2004). A multisite, randomized controlled trial for children with sexual abuserelated PTSD symptoms. Journal of the American Academy of Child \& Adolescent Psychiatry, 43(4), $393-402$. https://doi.org/10.1097/00004583-200404000-00005

Cohen, J. A., \& Mannarino, A. P. (1998). Interventions for sexually abused children: Initial treatment outcome findings. Child Maltreatment, 3(1), 17-26. https://doi.org/10.1177/1077559598003001002

Cohen, J. A., Mannarino, A. P., \& Deblinger, E. (2006). Treating trauma and traumatic grief in children and adolescents. Guilford Press. 
Cohen, J. A., Mannarino, A. P., \& Deblinger, E. (2017). Treating trauma and traumatic grief in children and adolescents (2nd ed.). Guilford Press.

Cohen, J. A., Mannarino, A. P., Kliethermes, M., \& Murray, L. A. (2012). Trauma-focused CBT for youth with complex trauma. Child Abuse \& Neglect, 36(6), 528 541. https://doi.org/10.1016/j.chiabu.2012.03.007

Cook, A., Blaustein, M., Spinazzola, J., \& van der Kolk, B. (2003). Complex trauma in children and adolescent. National Child Traumatic Stress Network (NCTSN), Complex Trauma $\quad$ Task force. https://www.nctsn.org/sites/default/files/resources/complex_trauma_in_children_and_adolescents.pdf

Finkelhor, D., Ormrod, R. K., \& Turner, H. A. (2007). Poly-victimization: a neglected component in child victimization. Child Abuse \& Neglect, 37(1), 7-26. https://doi.org/10.1016/j.chiabu.2006.06.008

Ford, J. D., \& Courtois, C. A. (2009). Treating complex traumatic stress disorders: An evidence-based guide. In C. A. Courtois, \& Ford, J. D. (Eds.), Treating complex traumatic stress disorders: An evidence-based guide (pp. 13-30). Guilford Press.

Hébert, M., \& Amédée, L. M. (2019, March). Diversity of profiles in child victims of sexual abuse: A latent class analysis. Communication au International Society for the Study of Trauma and Dissociation (ISSTD) 36th Conference, New York (NY), United States.

Hébert, M., \& Amédée, L. M. (submitted). Latent class analysis of post-traumatic stress symptoms and complex PTSD in child victims of sexual abuse and their response to Trauma-Focused Cognitive Behavioural Therapy.

Hébert, M., Cénat, J. M., Blais, M., Lavoie, F., \& Guerrier, M. (2016). Child sexual abuse, bullying, cyberbullying, and mental health problems among High school students: A moderated mediated model. Depression and Anxiety, 33(7), 623-629. https://doi.org/10.1002/da.22504

Hébert, M., \& Daignault, I. V. (2015). Challenges in treatment of sexually abused preschoolers: A pilot study of the effects of TF-CBT with preschool victims of sexual abuse in Quebec. Sexologies, 24(1), e21-e27. https://doi.org/10.1016/j.sexol.2014.09.003

Hébert, M., Moreau, C., Blais, M., Lavoie, F., \& Guerrier, M. (2017). Child sexual abuse as a risk factor for teen dating violence: Findings from a representative sample of Quebec youth. Journal of Child and Adolescent Trauma, 10(1), 51-61. https://doi.org/10.1007/s40653-016-0119-7

Hébert, M., \& Tourigny, M. (2015, November). A treatment outcome study of Trauma-Focused Cognitive Behavioral Therapy (TF-CBT) with a Quebec sample of sexually abused children. Communication au 31th Annual Meeting of the International Society for Traumatic Stress Studies (ISTSS), New Orleans (LA), United States.

Hodges, M., Godbout, N., Briere, J., Lanktree, C., Gilbert, A., \& Kletzka, N. T. (2013). Cumulative trauma and symptom complexity in children: A path analysis. Child Abuse \& Neglect, 3711), 891-898. http://doi.org/10.1016/j.chiabu.2013.04.001

Jensen, T. K., Holt, T., \& Ormhaug, S. M. (2017). A follow-up study from a multisite, randomized controlled trial for traumatized children receiving TF-CBT. Journal of Abnormal Child Psychology, 45(8), $1587-1597$. https://doi.org/10.1007/s10802-017-0270-0

Jensen T. K., Holt T., Ormhaug, S. M., Egeland K. E., Granly L. B., Hoaas L. E., \& Wentzel-Larsen T. (2014). A randomized effectiveness study comparing trauma-focused cognitive behavioral therapy with therapy as usual for youth. Journal of Clinical Child and Adolescent Psychology, 43(3), 356-369. https://doi.org/10.1080/15374416.2013.822307

Lanktree, C. B., \& Briere, J. (2017). Treating complex trauma in children and their families: An integrative approach. Sage Publications. https://doi.org/10.4135/9781071801291

Lewis, T., McElroy, E., Harlaar, N., \& Runyan, D. (2016). Does the impact of child sexual abuse differ from maltreated but non-sexually abused children? A prospective examination of the impact of child sexual abuse on internalizing and externalizing behavior problems. Child Abuse \& Neglect, 51, 31 40. https://doi.org/10.1016/j.chiabu.2015.11.016

Lyons, J. (2004). Redressing the emperor: Improving our children's public mental health system. Praeger.

Lyons, J. S., Griffin, E., Fazio, M., \& Lyons, M. B. (1999). Child and adolescent needs and strengths: An information integration tool for children and adolescents with mental health challenges (CANS-MH). Buddin Praed Foundation.

McMullen, J., O'Callaghan, P., Shannon, C., Black, A., \& Eakin, J. (2013). Group trauma-focused cognitive-behavioural therapy with former child soldiers and other war-affected boys in the DR Congo: A randomised controlled trial. Journal of Child Psychology and Psychiatry, 54(11), 1231-1241. https://doi.org/10.1111/jcpp.12094

Murray, L. K., Familiar, I., Skavenski, S., Jere, E., Cohen, J., Imasiku, M., Mayeya, J., Bass, J. K., \& Bolton, P. (2013). An evaluation of trauma focused cognitive behavioral therapy for children in Zambia. Child Abuse \& Neglect, 3712), 1175-1185. https://doi.org/10.1016/j.chiabu.2013.04.017

O'Callaghan, P., McMullen, J., Shannon, C., Rafferty, H., \& Black, A. (2013). A randomized controlled trial of trauma-focused cognitive behavioral therapy for sexually exploited, war-affected Congolese girls. Journal of the American Academy of Child and Adolescent Psychiatry, 52(4), 359-369. https://doi.org/10.1016/j.jaac.2013.01.013 
Runyon, M., Risch, E., \& Deblinger, E. 2019. Trauma-focused cognitive behavioral therapy. In L. J Farrell, T. H. Ollendick \& P. Muris, (Eds.), Innovations in CBT for childhood anxiety, OCD and PTSD: Improving access and outcomes (pp. 525-549). Cambridge University Press. https://doi.org/10.1017/9781108235655.026

Sachser, C., Keller, F., \& Goldbeck, L. (2016). Complex PTSD as proposed for ICD-11: Validation of a new disorder in children and adolescents and their response to trauma-focused cognitive behavioral therapy. Journal of Child Psychology and Psychiatry and Allied Disciplines, 58(2), 160-168. https://doi.org/10.1111/jcpp.12640

Tremblay-Perreault, A., Amédée, L. M., \& Hébert, M. (2017). Peer victimization in sexually abused children: Self-blame and posttraumatic stress symptoms as possible mechanisms. International Journal of Child and Adolescent Resilience, 5(1), 4-19.

van der Kolk, B. A., Pynoos, R. S., Cicchetti, D., Cloitre, M. Y., Ford, J. D., Lieberman, A. F., Putnam, F. W., Saxe, G., Spinazzola, J. Stolbach, B. C., \& Teicher, M. H. (2009). Proposal to include a developmental trauma disorder diagnosis for children and adolescents in DSM-V. https://pdfs.semanticscholar.org/7d01/b9cb0fdbf2f31bbc94a6846bb1e9064e2ace.pdf

Weiner, D. A., Schneider, A., \& Lyons, J. S. (2009). Evidence-based treatments for trauma among culturally diverse foster care youth: Treatment retention and outcomes. Children and Youth Services Review, 37(11), 1199-1205. https://doi.org/10.1016/j.childyouth.2009.08.013 